\title{
Right Hepatectomy, Open vs Laparoscopy: A Systematic Review
}

\author{
Anas Alhomaidhi, ${ }^{1,2}$ \\ ${ }^{1}$ Barts Cancer Institute (BCI), London, UK \\ ${ }^{2}$ University of London, London, UK \\ Email: dr.anas@hotmail.co.uk
}

Received July 11, 2012; revised August 14, 2012; accepted August 25, 2012

\begin{abstract}
Minimal access procedures show successes in various several medical fields. This issue brings a specific use of laparoscopy to the front to discuss in more detail which is the liver tumour resection. And, it's the high time for laparoscopy to be highlighted regarding to hepatobilliary system. It is significantly important to gain most of the benefits of the laparoscopy technology. Its advantages are already achieved in other operation type inside the body cavity such as cholecystectomy.
\end{abstract}

Keywords: Hepatectomy; Right Hepatectomy; Laparoscop; Laparotom; Liver Tumour

\section{Introduction}

“In 1929, Heinz Kalk, a German gastroenterologist, is considered the founder of the German School of Laparoscopy. Kalk developed a 135 degree lens system and a dual trocar approach. He used laparoscopy as a diagnostic method for liver and gallbladder disease. In 1939 he published his experience of 2000 liver biopsies performed using local anaesthesia without mortality [1].”

In general, hepatobilliary system has a various anatomical diversity. It also considered one of the highest organs body which affected by tumours. Since the liver receives the most toxic and deoxygenated blood from other body organs, it is highly likely that a tumour proceeds from its uncompensated cell during certain pathological abnormality. In addition, the liver is believed to be the most large-sized organ among other human body organs that is nearly one and a half kilogram in adult. It has an individual shape like wedge. Further, it has a unique consistency that gives the liver its architecture. It does not need reconstruction. Thus, it has such biological construction in which it allows self-regeneration process.

Accordingly, it became a hot topic today to explore the proper beneficial surgical approach to deal with hepatic tumour managements. An interested personal in dealing with liver tumour, either diagnostic or operative purposes, should bear in mind that the histological diversity, tumour extent and size, its anatomical location, and risk factors. Those points may help to produce an accurate standard method in liver tumour management involving its surgical resection. Although, today laparoscopic heaptectomy still has certain limitations. They are related to the patient's fitness, the used surgical technique, a properly skilled surgeon, and among others.

\section{Liver Hints}

\subsection{Hepatic Applied Anatomy}

Liver is considered as one of hepatobilliary systems which consist of liver, pancreas, spleen and gall bladder. All these organs integrated inside abdominal cavity in different anatomical region. However, liver and gall bladder locates in right upper quadrant of abdominal divisions. Pancreas exists nearly in epigastric region. And, there are linkages between all of these biliary tree organs that are portal vein, common bile duct, and arteries. Peritoneum folds in abdomen are covered whole liver surfaces except bare area which is on the posterior surface of liver itself, where inferior vena cava pass behind liver. Also, it covers in front of pancreas which is considered as retroperitoneal organ.

This peritoneal cover involves coronary ligament, which form left and right triangular ligament. The latter are the borders of liver bare area. Anteriorly, falciform ligament connects umbilicus with midline of liver. It has ligamentous teres in its lower border. Moreover, lesser omentum is attached by common bile duct, hepatic artery and portal vein.

Liver is divided by falciform ligament, ligamentom teres and ligamentom venosum to right and left lobe. Further, each lobe is divided to smaller anatomical region by hepatic veins and portal buds to segments (Figure 1).

There are eight segments. In one hand, left lobe con- 


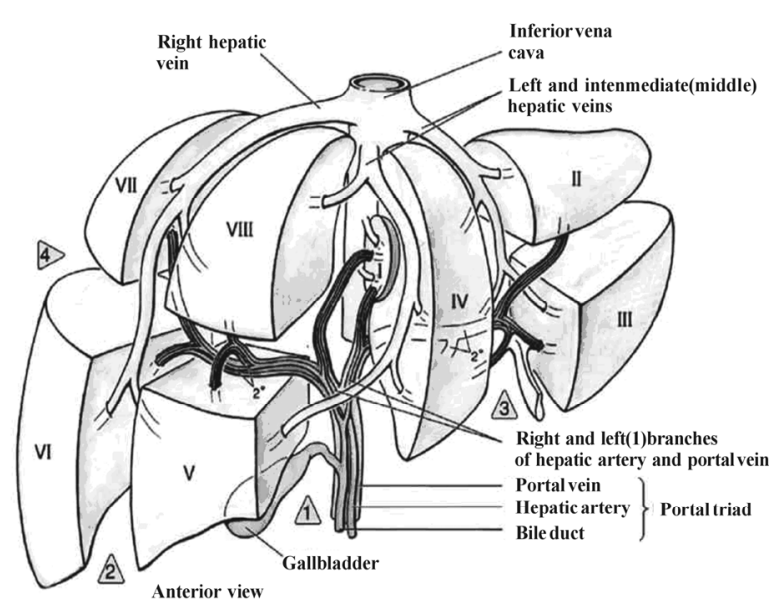

Figure 1. Liver segments.

sists of quadrate lobe, caudal lobe, and second and third segments. On the other hand, right lobe composes of fifth, sixth, seventh and eighth segments. Significantly, the prime importance to recognize and identify segments is partial liver resection for example low grade or confined tumor lesion. It gives the advantage of accuracy. Risky, Inferior Vena Cava locates between segment I and segment VII, where IVC is highly likely to be injured.

Liver hilum is known as "Porta Hepatis" that acts as a gate for vessels and ducts from and into the liver. It involves hepatic artery, portal vein, common hepatic duct and lymph nodes. Superiorly, liver is markedly closed to right diaphragm dome whereas it is diversely related to stomach in its left lobe and right kidney, duodenum and colon beneath right lobe inferiorly. Also, there is gall bladder fossa and in Porta Hepatis in its inferior aspect.

In general, liver is considered as the largest organ in size anatomically as well as the multifunctional body organ physiologically as it will be mentioned in details later.

In addition, under microscopic view, liver histological structure consists of acinar units. Each of these units involves 3 zones; a zone near arteriole, a zone near venule and middle zone. There are "Sinusoidal Spaces of Dess" separates each zone from another, where there is blood flow and microphages, "Kupffer cells".

\subsection{Hepatic Applied Physiology}

Liver has a multifunctional rule in body physiology. First, it contributes in fat, carbohydrates, and protein metabolisms. It forms a storage place for glucose and other essential elements such as Vitamin B12 and Iron. Secondly, in its excretion function there are many harmful substances that excreted by the live, for example ammonia, toxins and drugs, cholesterol, hormones, bacteria and foreign bodies. However, each of these deleterious particles has its specific excretion mechanism. Thirdly, liver is able to produce bile from half litre to one and a half of bile per day, thus gall bladder reserve it till it gets signal from stomach to drain it in duodenum to help in the digestion and absorption processes.

\subsection{Hepatic Applied Pathology}

Jaundice: Accretion of bilirubin in the body tissue due to its increase more than $35 \mu \mathrm{mol} / \mathrm{L}$ in serum. It reflects abnormal yellow colour which obviously appears on the eye sclera and the skin. However, causes of jaundice are divided to 3 main categories; pre-hepatic causes, hepatic, post-hepatic causes. For example; blood cell disorders, cirrhosis or HCC, and cholestatic conditions respectively. Cirrhosis: It is hepatic fibrosis that is caused by many known factors and it could be idiopathic too. Currently, it is believed to be the commonest reason to produce portal hypertension.

Tumour: Indeed, there are several known tumour might affect liver whether its parenchyma or as a cyst. Also, it is divided to two main categories benign and malignant. In one hand, the common malignant liver tumour is Hepatoma or Hepatocellular carcinoma, HCC. There are cholangiocarcinoma, hepatoblastoma and angiosarcom uncommonly cancerous liver tumours. On the other hand, there are many types of benign hepatic tumour that are highly likely to be found in female. In general, the trend of liver tumour resection laparoscopically is mostly preferable for benign tumour.

\section{Liver Resection}

\subsection{Open Hepatectomy}

Conventional therapy of the tumor that exists in liver through laparotomy is known for long time ago. In 1949, it was the first documented hepatectomy by Japanese surgeon called Ichio Honjo (Ryoichi Tsuchiya, 1988) [2]. Furthermore, this open technique uses a midline incision with subcostal incision where extended bilaterally. There are functional segment resection and anatomical segment resection. Clearly, the latter is preferable due to the bleeding limitation and prevention of the fistula formation as well as other positives. However, many facts about laparotomy hepatectomy surgery were intentionally omitted in this work science they are known by the majority and the world literature has already more than enough of its knowledge in detail, putting more effort to explore the next surgical generation.

\subsection{Laparoscopic Hepatectomy}

Initially, as part of the recent modern medical field especially in surgical techniques that are used in patient management with liver tumor, laparoscopic sunshine was raised in last few decades. However, early trial proce- 
dures of liver tumor cure laparoscopically were attempts in early 1990s (D. Bernard, et al., 2000) [3] and before. Further evidence is that "The first laparoscopic non anatomic resection of a Focal Nodular Hyperplasia (FNH) was reported by Gagner and colleagues in 1992, and this was followed by the first report of laparoscopic anatomic liver resection in 1996 (Edgar J. Figueredo, et al., 2008) [4]".

At that time, laparoscopic hepatectomy showed a promise in this new surgical technique hence it encouraged interested people to put more efforts to improve and to widen the liver resection experiences by performing more clinical trial, observation, and also by detailed researches.

Laparoscopic method is considered as one of the modern high technology utilities that needs as assessment as well as a comprehensive comparison to other conventional method. Further, it needs an analysis for its outcome efficacy and other critical measures related to any benefits would be gained from this new surgical concept. Fundamentally, laparoscopic method uses minimal invasion technique. This gives its usefulness and more advantages as a cure surgical treatment in tumour resection generally. Another point is that reliability and safety are significantly important for both the surgeon and the patients. Thereafter, specialized people focus on exploring those aspects regarding to certain technical measures which are followed to test hypothesis of the benefits of laparoscopy in liver tumour treatment. For this purpose and to prone the superiority of the laparoscopic type upon other available methods of tumour resection it needs a sufficient evidences beside an accuracy of the used methods in the analysis of the collected information. Moreover, data would be for or against the advantages of laparoscopy towered the differentiation between open and laparoscopy (Figure 2).

In addition, it is stated that it is important to discuss and explore all possible positives of tumour resection of

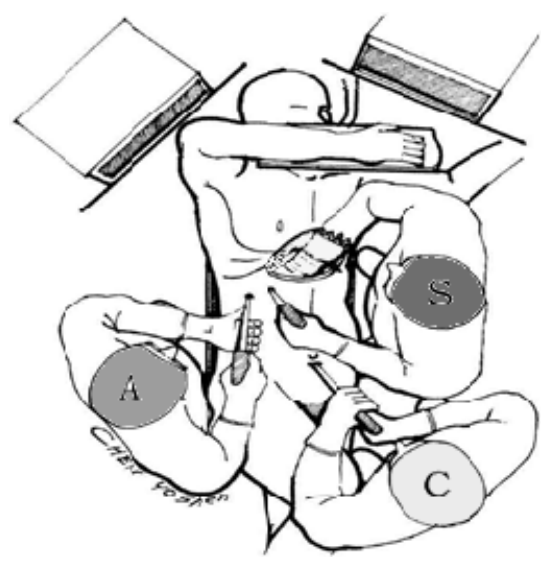

Figure 2. Laparoscopic personnel in-theatre positions $S$. Surgeon; A. Assistant surgeon; C. Cameraman. the liver, since cirrhosis incidence has being soared therefore increased tumour occurrence, by those invaluable instruments. It would be achieved by highly specialized surgeons as the liver has its unique anatomy beside its adjacent vital anatomical structures. In fact, certain measures of the operation outcomes are certainly wondering people who are dealing with tumours, specifically in the way how to resect it, the blood loss during and after the procedure and the operation time. They try to choose the most useful and feasible method to resect the targeted tumour for the patient's benefit and the surgeon's satisfaction. However, from the first attempt to resect a liver tumour using minimal invasive technique till today, instruments and devices, which are used, have being improved to meet the acceptable surgical standard and perhaps go further than so. In contrast, nowadays laparoscopic hepatectomy is still limited to some extent. These limitations are confined to some specific anatomical location of the liver, highly specialized trained personnel, and it is proper for particular level of local or distant extension of the liver tumour. It might be estimate this extension by the staging process in which laparoscopy has another function in surgery. Many practical procedures using laparoscopic technique including the following surgical skills:

- Staging

- Resection

- Thermal ablation.

- Cryosurgery.

- Cyst de-roofing

\subsubsection{Resections Approaches}

Initially, Minimal access procedure has converted the approach of many surgical operations, decreasing associated pain, operation time, hospital stay length, and achieving competitive results with open operations.

\subsubsection{Instruments}

Various types of instruments can be used to achieve the desire resection depend on multifactor as appear below in Figure 3. Surgical equipments required for laparo-

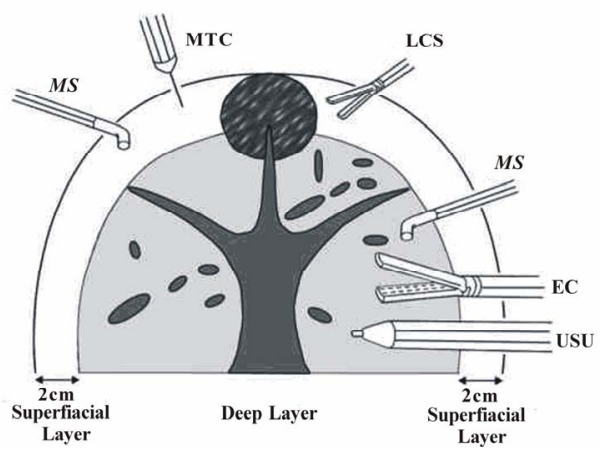

Figure 3. Laparoscopic instruments. 
scopic hepatectomy are: Microwave Tissue Coagu lator (MTC); Laparoscopic Coagulating Shears (LCS); Monopolar Sealer (MS); Endolinear Staplers (ELS); Ultrasonic Dissection (USU/UD); Endo Clinch Grasper (EC).

\subsubsection{Resection Indications}

Treatment of benign lesions is generally conservative. Surgery is considered primarily in cases where the tumour is causing significant abdominal pain, or if there is a high risk of rupture of the tumour. Furthermore, surgery should be done if the benign nature of the tumour cannot be confidently established, or if it is felt that the tumour has a risk of progression to a malignancy.

\section{Objectives}

The main objective of this thesis is to investigate, assess and analyse the safety, feasibility, reproducibility and surgical quality measures of the laparoscopy in comparison to conventional laparotomy on behalf of the liver tumour resection. Also, the subsidiary purpose involves overall outcomes to determine then extract any new advantages of laparoscopy over open method. Thus, to expose the advantages, difficulties and limitations of different available laparoscopic techniques those have been elicited in the published papers.

\section{Methodology}

This research underwent systematic review as a secondary research. It is cohort study type, comparing open and laparoscopic historical data from the world literature. Data collections source includes journals, periodic, text books, either from private sectors or public sectors including The British Library and QMUL's libraries; Bart's Library, Whitechapel Library and Mile End Library in London, United Kingdom. Another data gathering are from electronic data-base and other confidential website provenances such as PubMed, BMJ, BJS, Springer, springerlink, Googlescholar, World LaparoscopyHospital, Medline, Medscape, EMBASE, and ResearchGate.

Furthermore, it explores and makes the vision clearer about liver tumour resection. And, it is focusing on the effectiveness of each method, observing their outcome, survival rate, post operational hospital stay, and cosmetics. Another object, it illustrates the benefit versus the procedure cost itself and the post operation expenses. Lastly, it assesses the laparoscopic resection as safe as an open method by emphasizing on safety principles. This was a reviewing of particular data available from procedure have been performed till the time of this research will be produced. After previous paper collection and analysis processes, it will yield an end result by comparing laparoscopy to open surgical resection. Also there was a comprehensive reviewing for major issues th- roughout this research.

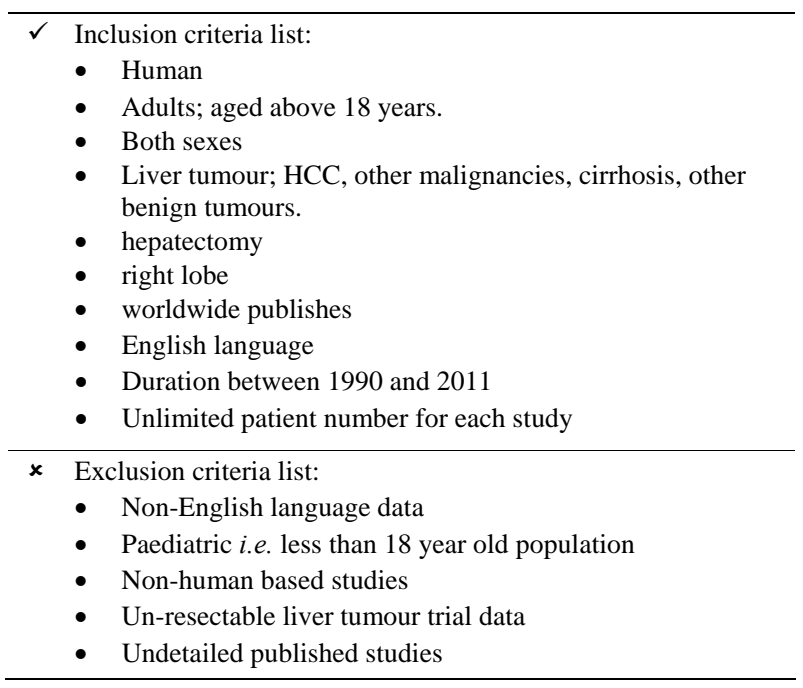

Additionally, numerical technique was used for dealing with each utilized study in this work. Hence, any published paper was included has a number which was given by the author of this thesis that referred to it. This is only for the ease purpose during the analysis section. In searching process, the star character $\left(^{*}\right)$ was being used in different medical journal engine search for all available suffix related to these thesis keywords. And, this research was conducted over ten months. It was supervised by three general surgeons including the head of cancer institution at Queen Mary, University of London and the degree director. Statistical advices were also gathered from a proficient statistician in the same medical institution.

\section{Abbreviations and Acronyms}

Referral abbreviations were been used in some sections. They are:

\begin{tabular}{ll}
\hline$\alpha-G T:$ & Alpha Glutamyl Transpeptidas \\
ALP: & Alkaline Phosphatase \\
ALT: & Alanine Aminotransferase \\
AST: & Aspartate Aminotransferase \\
CRC: & Colorectal Carcinoma \\
ELS: & Endolinear Staplers; \\
GA: & General Anaesthesia \\
HCC: & Hepatocellular Carcinoma \\
HS: & Hospital Stay \\
IVC: & Inferior Vena Cava \\
LA: & Local Anaesthesia \\
LCS: & Laparoscopic Coagulating Shears \\
LH: & Laparoscopic Hepatectomy \\
MS: & Monopolar Sealer \\
MTC: & Microwave Tissue Coagulator \\
OH: & Open Hepatectomy \\
USU/UD: & Ultrasonic Dissection \\
EC: & Endo Clinch Grasper \\
\hline
\end{tabular}




\section{Results}

From 1990 to 2010, 751 patients underwent open hepatectomy and 4207 patents underwent laparoscopic hepatectomy. There were 426 male adults and 325 female adults collectively. They were known their gender and 3628 mixed adults. Their age range was between 21 and 64 years who had laparoscopy and between 35 and 79 year old who had standard hepatectomy. However, 54 was the average age for the patients of both resection methods. Open procedures were performed in France, the USA, Taiwan, Chile, Korea, Japan, China, and Germany with 14, 100, 163, 66, 1, 23, 280, 49, and 55 patients respectively. These patient numbers were documented and were analysed in this research as its sample. And, however, for laparoscopy, operative experiences were carried out in France, Italy, the Netherlands, Japan, Taiwan, Korea, Australia, china, the UK, the USA, Brazil, and Singapore with 52, 39, 50, 502, 123, 240, 28, 25, 40, 31,1 , and 5 patients respectively.

Tumour sizes which been resected with open surgery ranged from $33.5 \mathrm{~mm}$ to $150 \mathrm{~mm}$ whereas other resection method was used to dissect tumour that sized between < $10 \mathrm{~mm}$ and $50 \mathrm{~mm}$. Operating time was 291 minutes for $\mathrm{OH}$ (191 - 481 minutes; range) and 229 minutes for LH (115 - 435 minutes; range).

Mean blood loss in LH was $260 \mathrm{ml}$ and in $\mathrm{OH} 1290 \mathrm{ml}$. The minimum blood loss was $839 \mathrm{ml}$ which was higher than the laparoscopic procedure average of blood loss and maximum was $1325.9 \mathrm{ml}$.

In our sample, Complications were existed in $44.8 \%$ in $\mathrm{OH}$ and $28.3 \%$ in $\mathrm{LH}$ as percentages of chosen total number. That's roughly shows lesser post-operative complications with laparoscopic use.

Mortality was low in both excision approaches. In $\mathrm{OH}$ and $\mathrm{LH}$ were the recorded mortality in literature as $0.42 \%$ and $0.37 \%$ respectively. It indicates slight deference in between.

Patients who underwent $\mathrm{OH}$ needed 8 to 30 days to stay in hospital (mean: 14.8 days), meanwhile, those who had LH stayed 3 to 15.3 days in hospital (mean: 8.4 days) postoperatively.

\section{Figures}

\subsection{Operating Time}

After data collection, they went throughout processing stage via the way to fit the purpose of this work. There was analysing them. Analysing process yielded the difference between laparoscopy and laparotomy in liver tumour managements. However, a statistical Chart 1 graph has been used to assess that difference. It illustrates that laparoscopic hepatectomy had less operating time by 65 minutes and 13 seconds in average than open Hepatectomy that been used associated with laparotomy to

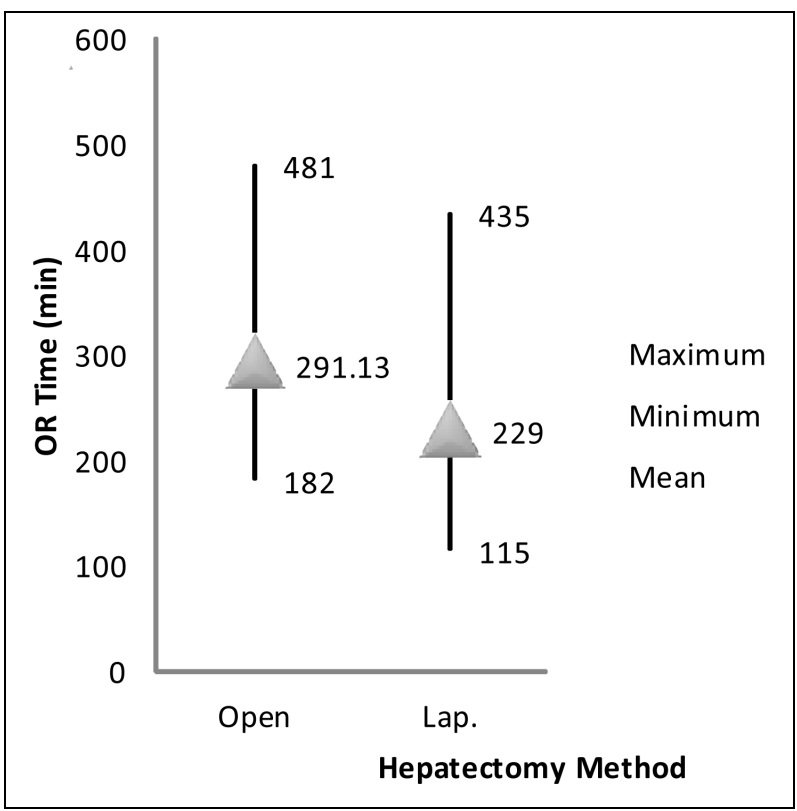

Chart 1. Operating time comparison.

access the abdominal cavity. In another way, laparoscopy takes less time to resect a liver tumour by more than an hour. Moreover, maximum and minimum operating time have been recorded up to date confirm the nearly the same difference in superiority to the minimal access procedure either.

In one hand, the longest operation to take a tumour from the liver out was about 481 minutes (Junji Ueda, et al.) [5] for open dissection method. That was as a published recorded. And, for laparoscopic operative duration, it has been published around 435 minutes (Kyung-Suk Suh, et al.) [6]. In another hand, the shortest recorded durations for both open and laparoscopic resections were 182 (Alexis Laurent, et al.) [7] and 115 minutes (J. L. Dulucq, et al.) [8], respectively. Together, using minimal invasion access to manipulate liver tumour surgically has the superiority advantage over the conventional method regarding the time aspect.

\subsection{Blood Loss}

Significantly, bleeding during a surgical procedure is one of the most concerning problem for any surgical worker. It still happened even with existed high technological monitoring protocols and operating skills.

Further, human blood is believed to be the most expensive fluid in life. It is invaluable by all meanings. It is needed to be saved as much as possible. However, surgeons put their effort inside theatre rooms to minimize inevitable blood loss intra-operatively, preventing blood from going outside vessels by using meticulous surgical techniques. They keep searching for the best method to approach the human body cavities to manipulate its or- 
gans. Therefore, this is as a part of patient's managements either for investigational or treating purposes.

However, blood loss while liver tumour resection is inescapable. There are two known approaches; the conventional access and the minimal access methods to do so. In this systematic review, both of them were reviewed in detail in the previous world published papers. Those were recorded their intra-operative blood loss measures. Thereafter, they were collected, processed and then were compared laparoscopy to laparotomy. Next, cylindrical bar Chart 2 graph was utilized for the contrast horizontally. After, it yielded that open hepatectomy associated with $1290 \mathrm{ml}$ of blood volume that been lost during the resection whereas laparoscopic hepatectomy needed as little as $260 \mathrm{ml}$ only in average for whole performance. In Masato Nagino, et al. [9] was maximum intra-operative blood loss that was $2354 \mathrm{ml}$ and $648 \mathrm{ml}$ was the minimum loss of blood was needed to resect tumour with laparotomy. Using laparoscopy could lose blood as minimum as 104 $\mathrm{ml}$ (S. Laura, et al.) [10]. As a result, of the comparison one can notices that a litre of blood was conserved as a difference between the two methods. Eventually, laparoscopic is saving patient's blood which always in shortage.

\subsection{Morbidity}

Post-operative morbidity was relatively low in both open and laparoscopy collections. They included complications such as; ascites and pleural effusion, bile leakage, wound infection and sepsis, abscess or fistula formation, bleeding, liver failure, anaphylactic shock and among others. In open hepatectomy, $\mathrm{OH}$, patients had at least one of those documented complications as a direct result of the resection procedures were represented by $44.8 \%$ of the total registered patients as Chart $\mathbf{3}$ shows.

In contrast, only $28 \%$ of those patients whom underwent laparoscopic hepatectomy, pointed out as "LH" on the sidebar chart, had documented morbidity after having minimal invasive procedure. Together, it confirms that the superiority in light of liver resection is for the LH as

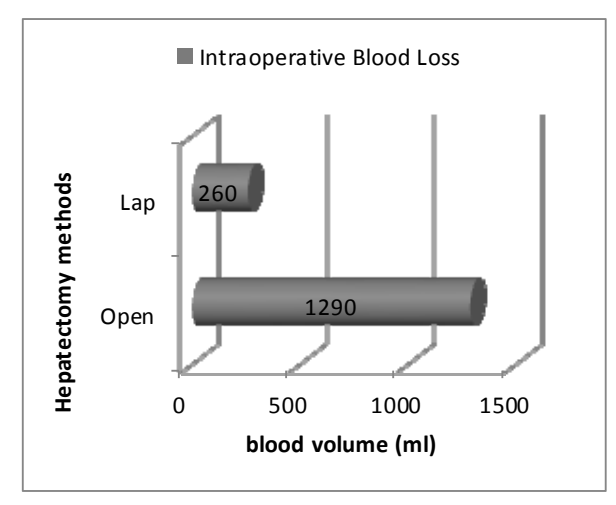

Chart 2. Intraoperative blood loss. it keeps the patient's health stable by reducing the surgical risks post-operatively.

\subsection{Mortality}

Firstly, another surgical measure to assess each approach quality is the number of deaths within 30 days from operation, which was considered in this systematic review. However, laparoscopic techniques dramatically reduced the mortality rate due to in-theatre causes. It feasibly became like a routine procedure on the everyday schedule. In fact, Laparoscopic is based on minimal invasive concept as it was mentioned above hence it minimize the iatrogenic causes of deaths. This study confirmed this fact by comparing the rate of deaths, mortality, for both conventional approach as a standard liver tumour resection and the modern laparoscopic resection.

In addition, the conical graph in this particular section, Chart 4, confirms by evidence that support the theory of laparoscopic advantages over other compare method. Also, it demonstrates that the proportion of 42 patients died after having tumour dissection of the liver in 10,000 patients whom underwent laparotomy. For instance, in

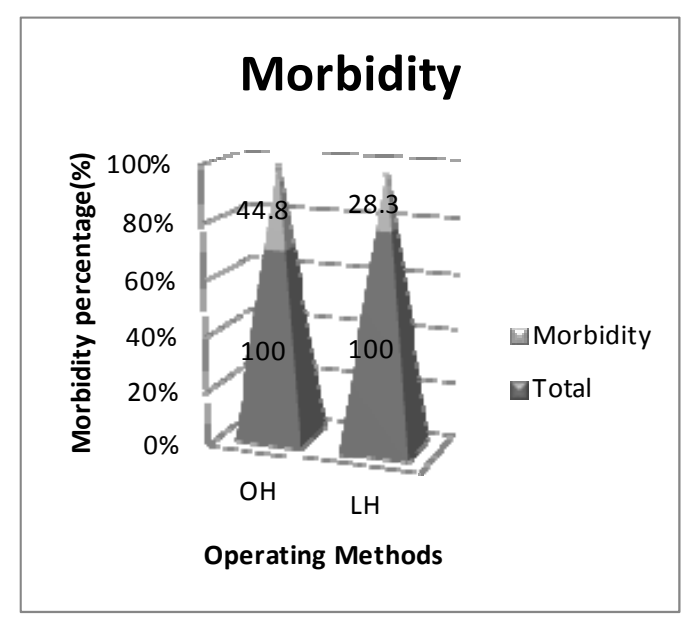

Chart 3. Morbidity comparison.

\section{Mortality}

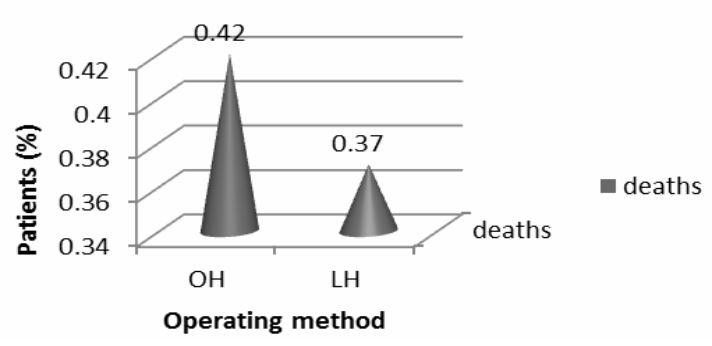

Chart 4. Mortality comparison. 
Alexis Laurent, et al. [11]. 2 patients out of 14 died due to open liver resection, and in G. Bhargavi, et al. [11]. 4 out of 100 patients were the documented mortality. However, 37 in 10,000 as mortality rate due to having laparoscopic dissection. For example, 1 patient out of 16 died because of LH (B. Giulio, et al.) [12]. Ultimately, it means that 5 patients' lives were saved by using minimal access procedure inside operating room.

\subsection{Hospital Stay}

Generally, laparoscopy is known as a minimal access approach into abdominal cavity. It uses small calibre ports via abdominal wall. They are ranged from $5 \mathrm{~mm}$ and $15 \mathrm{~mm}$ in diameter. Thus, its wound of abdominal wall heals earlier rather fewer accompanied wound complications. Therefore, it needs less hospital wound care. However, previous published observations have confirmed the fact. Hospital stay was recorded in many surgical occasions. Hospital stay reflects the duration that starts after the end of a surgical operation until the start of the patient's discharge from hospital.

Introducing laparoscopic interventions in surgical field has dramatically shortened the necessary stay in hospitals. Hence, increases the advantages of being recovered earlier. In Chart 5, vertical lines were used to construct the hospital stay comparison graph. Then, it was integrated in this section of the study to determine this particular positive aspect that belonging to laparoscopic use. It has both maximum and minimum periods in which post-hepatectomy patients were operate them in hospital. It, however, implies the mean of hospital stay post-operatively which was statistically significant. 30 and 15 days were the maximum hospital stay for laparotomy (Zenichi Morise, et al.) [13] and laparoscopy (Alexis Laurent, et al.)

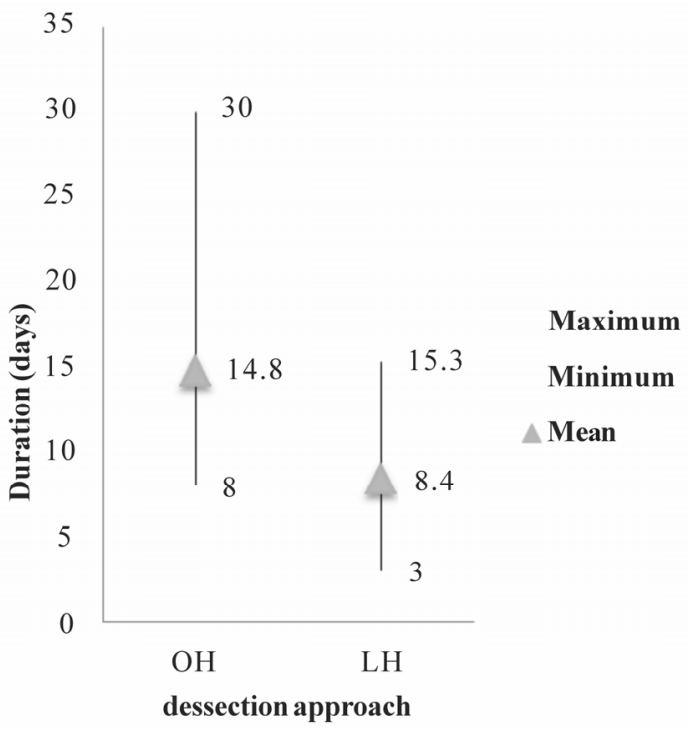

Chart 5. Hospital stay comparison. respectively. And, 7 and 3 days were the shortest documented hospital stay for open (K. F. Lee, et al.) [14] and laparoscopy (S. Laura, et al.) respectively. Surprisingly, it elicits that the average of laparoscopic hospital stay was roughly half of the other compare approach. Subsequently, performing laparoscopic resection saved half of the cost of hospital stay whether for the patient or the hospital budget. It gives an opportunity for more patients to benefit from surgical services.

\section{Future}

Laparoscopic hepatectomy is predicted to improve and develop in incoming years nonetheless it shows certain degree of obstacles nowadays. Updated results and overall technology improvements will be a great motivation for its development. Mini-invasive Liver resection needs further clinical trials and more studies. It needs to be refined for wider uses. Thereby, the range of the selection criteria can be broader. Cost superiority over standard method is recommended issue for future study.

\section{Acknowledgements}

The author confirms complete of interest as this work targets specialized surgeons and interested individuals.

\section{REFERENCES}

[1] The History of Laparoscopy, 1996. http://www.laparoscopy.com/shows/lapstry6.htm

[2] R. Tsuchiya, “A Tribute to the Memory of Dr. Ichio Honjo,” Vol.19, No. 1, 1989, p. 1.

http://www.springerlink.com/content/t07454383j9760t0/ doi:10.1007/BF02471559

[3] B. Descottes, F. Lachachi, M. Sodji, D. Valleix, S. Durand-Fontanier, B. Pech, D. Laclause and D. Grousseau, "Early Experience with Laparoscopic Approach for Solid Liver Tumors: Initial 16 Cases," Annals of Surgery, Vol. 232, No. 5, 2000, pp. 641-645.

http://www.ncbi.nlm.nih.gov/pmc/articles/PMC1421217/ doi:10.1097/00000658-200011000-00004

[4] E. J. Figueredo and R. S. Yeung, "Laparoscopic Liver Resection,” The Medscape Journal of Medicine, Vol. 10, No. 3, 2008, p. 68.

http://www.medscape.com/viewarticle/568596

[5] J. Ueda, H. Yoshida, Y. Mamada, N. Taniai, S. Mineta, M. Yoshioka, A. Hirakata, Y. Kawano, T. Kanda and E. Uchida, "Resection of Hepatocellular Carcinoma Recurring in the Diaphragm after Right Hepatic Lobectomy," Journal of Nippon Medical School, Vol. 78, No. 1, 2011, pp. 30-33.

http://www.jstage.jst.go.jp/article/jnms/78/1/78_30/_article

[6] S. Kyung-Suk, Y. Nam-Joon, T. Kim, J. Kim, W. Y. Shin, H. W. Lee, H.-S. Han and K. U. Lee, "Laparoscopy-Assisted Donor Right Hepatectomy Using a Hand Port Sys- 
tem Preserving the Middle Hepatic Vein Branches,” World Journal of Surgery, Vol. 33, No. 3, 2009, pp. 526533, http://www.springerlink.com/content/547n4472h4k3wj04/ doi:10.1007/s00268-008-9842-z

[7] A. Laurent, D. Cherqui, M. Lesurtel, F. Brunetti, C. Tayar and P.-L. Fagniez, "Laparoscopic Liver Resection for Subcapsular Hepatocellular Carcinoma Complicating Chronic Liver Disease,” Archives of Surgery, Vol. 138, No. 7, 2003, pp. 763-776,

http://archsurg.ama-assn.org/cgi/reprint/138/7/763.pdf doi:10.1001/archsurg.138.7.763

[8] J. L. Dulucq, P. Wintringer, C. Stabilini, J. Berticelli and A. Mahajna, "Laparoscopic Liver Resections: A Single Center Experience,” Surgical Endoscopy and Other Interventional Techniques, Vol. 19, No. 7, 2005, pp. 886-891. http://www.springerlink.com/content/t4h4311872w46477/

[9] M. Nagino, J. Kamiya, T. Arai, H. Nishio, T. Ebata, and Y. Nimura, “Anatomic Right Hepatic Trisectionectomy (Extended Right Hepatectomy) with Caudate Lobectomy for Hilar Cholangiocarcinoma," Archives of Surgery, Vol. 243, No. 1, 2006, pp. 28-32.

http:/www.ncbi.nlm.nih.gov/pmc/articles/PMC1449975/ doi:10.1097/01.sla.0000193604.72436.63

[10] S. Laura, M. S. Metcalfe, A. D. Strickland, E. J. Elsey, G. S. Robertson and D. M. Lloyd, "Lessons from Laparoscopic Liver Surgery: A Nine-Year Case Series,” HPB Sur- gery, Vol. 2008, No. 2008, 2008, 6 p. http://www.ncbi.nlm.nih.gov/pmc/articles/PMC2495020/

[11] B. Gali, J. Y. Findlay, D. J. Plevak, C. B. Rosen, R. Dierkhising and D. M. Nagorney, "Right Hepatectomy for Living Liver Donation vs Right Hepatectomy for Disease,” Archives of Surgery, Vol. 142, No. 5, 2007, pp. 467472. http://archsurg.ama-assn.org/cgi/reprint/142/5/467.pdf doi:10.1001/archsurg.142.5.467

[12] G. Belli, C. Fantini, A. D’Agostino, A. Belli, and N. Russolillo, "Laparoscopic Liver Resections for Hepato-Cellular Carcinoma (HCC) in Cirrhotic Patients," HPB (Oxford), Vol. 6, No. 4, 2004, pp. 236-246.

http://www.ncbi.nlm.nih.gov/pmc/articles/PMC2020680/

[13] Z. Morise, K. Yamafuji, T. Takahashi, A. Asami, K. Takeshima, N. Hayashi, H. Baba, T. Endo and Y. Tokura, "Hepatic Resection for Colorectal Metastases in the Caudate Lobe of the Liver," Journal of Hepato-Biliary-Pancreatic Surgery, Vol. 11, No. 5, 2004, pp. 348-351. http://www.springerlink.com/content/kyevp3q914mw8k1b/

[14] K. F. Lee, Y. S. Cheung, C. N. Chong, Y. Y. Tsang, W. W. Ng, E. Ling, J. Wong and P. B. Lai, "Laparoscopic versus Open Hepatectomy for Liver Tumours: A Case Control Study," Hong Kong Medical Journal, Vol. 13, No. 6, 2007, pp. 442-448.

http://www.hkmj.org/abstracts/v13n6/442.htm 